\title{
Signs of neuroinflammation outweigh neurodegeneration as predictors for early conversion to MS
}

\author{
Nervana Mohamed El Fayomy', Hanan Helmy Mohamed', Manal Mohamed Kamal², Aya Hamid Alsayyad', \\ Enas Hamid Alsayyad ${ }^{1}$ and Reham Mohamed Shamloul ${ }^{1 *}$ (D)
}

\begin{abstract}
Background: The pathophysiological mechanisms underlying multiple sclerosis include both inflammatory and degenerative processes. We aimed to study and compare markers of neuroinflammation and neurodegeneration in patients with first presentation of demyelinating disorder and to prospectively identify which of the studied markers serve as predictors for early conversion to multiple sclerosis. Thus, 42 patients with first clinical manifestations suggestive of demyelinating disease were included in a prospective study. Subjects underwent thorough history taking and clinical evaluation. Laboratory studies involved analysis of cerebrospinal fluid (CSF) and serum chitinase 3-like 1 levels. Brain imaging included MRI and ultrasonographic assessment.
\end{abstract}

Results: T1 black holes, elevated oligoclonal bands (OCB), high baseline T2 lesion load, and enhanced MRI lesions were significantly higher in patients with 1st attack multiple sclerosis. Significantly higher CSF-OCB and serum chitinase 3-like 1 protein was detected in patients with multiple sclerosis (MS) compared to clinically isolated syndrome, and higher levels in MS convertors than non-convertors. Cognitive dysfunction evaluated by MoCA test and brain atrophy assessed using transcranial sonography did not show significant difference among the studied groups. Logistic regression analysis showed that heavy T2 lesion load served as the only predictor of conversion to MS.

Conclusion: Early conversion to MS after first attack of demyelination is related to detection of signs of neuroinflammation rather than neurodegeneration.

Keywords: Multiple sclerosis, CIS, Prognostic markers, Neuroinflammatory, Neurodegenerative

\section{Background}

Attempts to pursue novel markers that would not only predict which patient with clinically isolated syndrome (CIS) will convert to clinically definite multiple sclerosis (CDMS) but also the disease stage, severity and possible development of disability are still required especially in early stages as this will strongly impact the MS management and treatment strategies $[1,2]$.

\footnotetext{
* Correspondence: reham.shamloul@kasralainy.edu.eg

1 Neurology Department, Cairo University, Cairo, Egypt

Full list of author information is available at the end of the article
}

Multiple variables including clinical picture, imaging protocols, and other para-clinical tests as CSF studies have been evaluated as prognostic factors [1] all of which addressing either signs of neurodegeneration or inflammation of the disease.

Several studies reported that early axonal degeneration and brain atrophy may predict short time for conversion to CDMS [3]. The presence of cognitive impairment was a measure that reflects early neuronal degeneration in some studies [4]; moreover, recent but fewer studies used parenchymatous transcranial sonography to visualize the brain parenchyma and ventricular system as a measure of brain atrophy in multiple sclerosis (MS)

\section{Springer Open}

(c) The Author(s). 2021 Open Access This article is licensed under a Creative Commons Attribution 4.0 International License, which permits use, sharing, adaptation, distribution and reproduction in any medium or format, as long as you give appropriate credit to the original author(s) and the source, provide a link to the Creative Commons licence, and indicate if changes were made. The images or other third party material in this article are included in the article's Creative Commons licence, unless indicated otherwise in a credit line to the material. If material is not included in the article's Creative Commons licence and your intended use is not permitted by statutory regulation or exceeds the permitted use, you will need to obtain permission directly from the copyright holder. To view a copy of this licence, visit http://creativecommons.org/licenses/by/4.0/. 
patients. Muller and colleagues [5] found that third ventricular enlargement is associated with increased risk for future MS relapses while Walter and colleagues [6] reported that hyperechogenicity of basal ganglia (BG) and substantia nigra (SN) due to trace metal (mainly iron) accumulation in MS patients is associated with disease progression.

On the other hand, several markers of inflammation have been studied as predictors for conversion to CDMS; of those, the most significant were the presence of CSF oligoclonal bands [7] and a new biomarker chitinase 3-like 1 (CHI3L1) [8]. CHI3L1 is a normal candidate of human serum which plays a role during inflammation and tissue remodeling. It is upregulated in different conditions with chronic inflammation [9]; thus, it is not specific for MS. However several studies reported that it may act as a marker for conversion from CIS to MS [10] and also as marker for disease progression at time of diagnosis [11].

To date, magnetic resonance imaging (MRI) is still the most important predictor for early conversion to CDMS, whether via detection of signs of inflammation as abnormal baseline MRI or enhancing lesions [12] or neurodegeneration as presence of $\mathrm{T} 1$ black holes and/or brain atrophy.

In this study, we aimed to investigate and compare different clinical, laboratory (biochemical) and brain imaging (using transcranial sonography and MRI) findings related to neurodegenerative and neuroinflammatory processes in patients with CIS and first attack of MS and detect their value as predictors for early conversion from CIS to MS.

\section{Methods}

This is a prospective observational cohort study that included 42 patients presented with first clinical manifestations suggestive of demyelinating disease. The study was conducted over a period of 2 years and consecutive patients from both sexes attending the outpatient clinic of Neurology department were enrolled if their age ranged from 18 to 50 years, had no better explanation of his/her neurological event, and had the ability to tolerate the investigations or clinical assessment (patients with severe visual affection, unable to read/write, or with severe weakness were excluded).

All patients were subjected to full neurological evaluation and assessed using Expanded Disability Status Scale (EDSS) [13]. According to the functional systems involved, the patients were categorized as monofocal/ multifocal presentation [14]. Cognitive assessment was done using the Arabic version of Montreal Cognitive Assessment (MoCA) test to detect mild cognitive impairment [3], validated in 2018 [15].
Serum samples were withdrawn for laboratory assessment to exclude better explanation as complete blood picture, liver functions, kidney functions, ESR, thyroid function tests, vasculitic profile as antinuclear antibody (ANA), antineutrophil cytoplasmic antibodies (ANCA), anti-Ds DNA, anti-cardiolipin antibodies, lupus anticoagulant, tumor markers, and serum aquaporin 4 (if needed). Serum chitinase 3-like 1 protein (CHI3L1) samples were collected from patients at least 28 days from the onset of the clinical attack in 3-milliliter $(\mathrm{ml})$ blood samples withdrawn on plain tubes. The samples were allowed to clot and sera were separated and put at -20 ${ }^{\circ} \mathrm{C}$ until assay. Chitinase 3-like 1 was measured by enzyme immunoassay technique using Rand D kit, Lot P126705 purchased from R and D systems. The CSFoligoclonal bands (OCB) and IgG index were collected from patient's data.

Transcranial sonography (TCS) was used to assess brain parenchyma by Philips IU22 machine, using phased array (2-3.5 MHZ) and linear probe (MHZ) respectively, through trans-temporal and trans-orbital approach respectively. Patients were placed in supine position, with examiner on patient's head; room was darkened. TCS was done through trans-temporal approach parallel to orbito-meatal line; 2 standard planes were assessed, first the mesencephalic plane for visualization of midbrain structures as substantia nigra (after visualization of the brain stem, resembling a "butterfly" shape, the picture was frozen and then enlarged by $2-3$ times. After manual tracing of the substantia nigra, planimetric evaluation of its area was performed the average of 3 planimetric measurements was taken). Values were then classified as normal, hyperechogenic, and hypoechogenic. Secondly, the diencephalic plane was assessed for measurement of width of third ventricle and frontal horn of lateral ventricle (normal or dilated). At thalamus level, after tilting the ultrasound beam by approximately $10^{\circ}$ up from the position of the mesencephalon, evaluation was related to the diameter of the third ventricle and the width of the frontal horn of the lateral ventricle on the opposite side. We used the Egyptian cut-off values [16], the unilateral substantia nigra measured surface area of echogenicity was considered normal if $\leq 0.19 \mathrm{~cm}^{2}$, the bilateral sum of substantia nigra measured surface area of echogenicity was considered hypo-echoic if $\leq 0.15 \mathrm{~cm}^{2}$, and third ventricle diameter was considered dilated if $\geq 0.23 \mathrm{~cm}$. The left frontal horn of lateral ventricle was considered dilated if $>0.37 \mathrm{~cm}$, and the right frontal horn of lateral ventricle was considered dilated if $>0.36 \mathrm{~cm}$. TCS examinations were performed by 2 independent investigators who were blinded to the clinical data of the patients. A structure was only regarded as abnormal on TCS if the findings of both investigators agreed. 
Brain imaging was done for all patients at presentation, on Philips Interna 1.5T, Philips, Achieva 1.5T and GE signa $0.2 \mathrm{~T}$ systems, axial $\mathrm{T} 1, \mathrm{~T} 2$, and FLAIR, and sagittal T2/FLAIR, post contrast axial and sagittal T1, and MRI spinal cord (was done when needed) (cervical cord or other levels, either with contrast or not). It was assessed for lesions typical or atypical for MS, dissemination in space (DIS), and dissemination in time (DIT) according to the 2010 revised McDonald criteria [17]. All MRI assessments were performed by a single rater blinded to clinical data.

The 42 recruited patients were then classified into two groups, patients with clinically isolated syndrome (group 1 ), who presented with first demyelinating event not fulfilling 2010 revised McDonald criteria [17], and patients with first attack of multiple sclerosis (group 2) who presented with first demyelinating event fulfilling 2010 revised McDonald criteria. CIS patients were followed for 1 year for appearance of new symptoms or worsening of already present symptoms lasting at least for $24 \mathrm{~h}$. Follow-up MRI brain was done at 6 and 12 months; after follow-up period, patients were classified into two groups, patients who remained as CIS (group of MS non-convertor) and patients who converted to MS (McDonald criteria 2017 criteria for MS diagnosis [17] (group of MS convertor).

Signs of neuroinflammation assessed included CSF OCB, IgG index, Chitinase 3-like 1 protein (CHI3L1), brain MRI T2 lesion load, and enhancing white matter lesions, while signs of neurodegeneration evaluated included cognitive dysfunction assessed by MOCA, markers of brain atrophy detected by parenchymatous transcranial sonography, hyper-echogenicity of SN and or dilated third ventricle and frontal horn of lateral ventricle, and T1 black holes in brain MRI.

\section{Statistical analysis}

Data were coded and entered using the statistical package SPSS the Statistical Package of Social Science Software program (IBM SPSS Statistics for Windows, Version 23.0. Armonk, NY: IBM Corp.). Data was summarized using mean, standard deviation, median, minimum, and maximum in quantitative data and using frequency (count) and relative frequency (percentage) for categorical data. Comparisons between quantitative variables were done using the non-parametric KruskalWallis and Mann-Whitney tests. For comparing categorical data, chi-square $\left(x^{2}\right)$ test was performed. Exact test was used instead when the expected frequency is less than 5. Logistic regression was done to detect independent predictors of conversion. $P$-values less than 0.05 were considered as statistically significant. All participants in the study provided informed verbal consent.

\section{Results}

We identified forty-two patients presented with first clinical manifestations suggestive of demyelinating disease, twenty-two patients (52.4\%) with clinically isolated syndrome (CIS) and twenty patients (47.6\%) with first attack of multiple sclerosis (MS). Detailed assessment of the patients' clinical presentation, cognitive affection, and EDSS are presented in Table 1. Only fifteen (35.7\%) patients accepted to do CSF analysis, 5 patients (25\%) of MS group, and 10 patients $(45.5 \%)$ of CIS group while serum CHI3L1 level was done for 38 patients (3 patients refused to give their blood sample without providing a reason); data are shown in Table 2. Transcranial sonography (TCS) and brain MRI were done to all patients; the results are presented in Table 3.

During the follow-up period, nine patients (40.9\%) of CIS group converted to MS, the mean time for conversion was $6.75 \pm 3.24$ months, 5 patients $(55.6 \%)$ developed new attack and diagnosed as having clinically definite MS, and 4 patients (44.4\%) had changes only in MRI in the form of appearance of new lesions compared to baseline MRI, but without any new clinical event, so diagnosed as McDonald MS.

Logistic regression was done to detect independent predictors of conversion; the suggested predictors were MoCA test, CHI3L1 assay, ultrasound ventricular diameter, and MRI total number of lesions. After removal of the confounder factors, MRI total number of lesions was the only predictor of conversion of CIS to MS patients (Table 4).

MS multiple sclerosis, $O R$ odds ratio, $C I$ confidence interval, $p>0.05=\mathrm{NS},{ }^{*} p<0.05=$ significant

\section{Discussion}

Comparing signs of inflammation and degeneration between patients with CIS and CDMS patients and evaluating their predictive value to detect conversion of CIS patients to MS has not been previously studied. Such issue remains of great importance so as to decide whether to start treatment with disease modifying therapy (DMT) or not [18].

Clinical characteristics showed that multifocal presentation was significantly higher in the MS group as well as monofocal sensory presentation compared to CIS patients; however, none of these presentations differed between the MS convertors and non-convertors.

In this study, on assessing signs of inflammation, it was found that the percentage of patients with OCB was statistically higher in MS converter (100\%) than non-converter group (25\%), which goes with previous studies that reported positive CSF OCB at time of CIS was a strong predictor for conversion to MS [7, 18]. Also another marker, chitinase 3 -like 1 protein (CHI3L1) was significantly higher in the MS compared to CIS patients, and higher in 
Table 1 Clinical characteristics of all studied groups

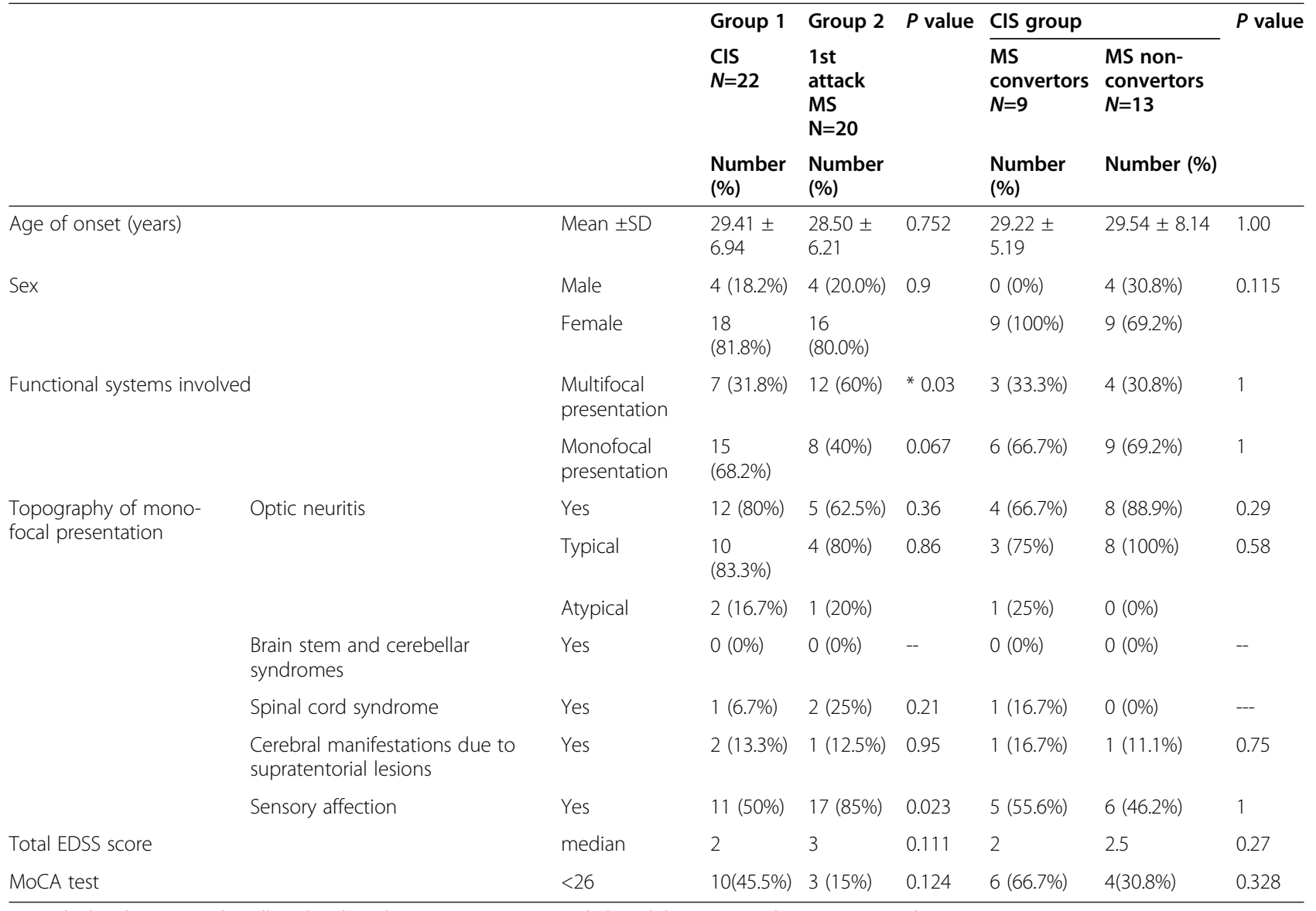

MS multiple sclerosis, CIS clinically isolated syndrome, EDSS using Expanded Disability Status Scale, MoCA Montreal Cognitive Assessment ${ }^{*} p<0.05=$ significant

MS convertors than non-convertors when comparing the mean values, but such significance was lost upon comparing median values. Previous studies also show inconsistent results regarding the predictive value of CHI3L1 levels; Hinsinger and colleagues [11] found that increased serum and CSF CHI3L1 in CIS patients was associated with increased risk for conversion to MS while another study by
Comabella and colleagues [8] showed that serum CHI3L1 was not associated with increased risk for conversion in contrast to its CSF level; thus, we can conclude that serum CHI3L1 may have a possible role in prediction of MS conversion but requires further confirmation.

On the other hand, magnetic resonance imaging (MRI) revealed that the number of patients with typical

Table 2 Laboratory results of the studied groups

\begin{tabular}{|c|c|c|c|c|c|c|}
\hline \multirow[t]{3}{*}{ Lab test } & \multirow{3}{*}{$\begin{array}{l}\text { Group } 1 \\
\text { CIS } \\
N=22 \\
\text { Number (\%) }\end{array}$} & \multirow{3}{*}{$\begin{array}{l}\text { Group } 2 \\
1 \text { st attack MS } \\
N=20 \\
\text { Number (\%) }\end{array}$} & \multirow[t]{3}{*}{$p$-value } & \multicolumn{2}{|l|}{ CIS group } & \multirow[t]{3}{*}{$p$-value } \\
\hline & & & & \multirow{2}{*}{$\begin{array}{l}\text { MS convertors } \\
N=9 \\
\text { Number (\%) }\end{array}$} & \multirow{2}{*}{$\begin{array}{l}\text { MS non-convertors } \\
N=13 \\
\text { Number (\%) }\end{array}$} & \\
\hline & & & & & & \\
\hline Serum chitinase3 like $1 \mathrm{pg} / \mathrm{ml}^{\#}$ mean & 30237.06 & 55663.68 & ${ }^{*} 0.038$ & 42347.1429 & 29328.3333 & ${ }^{*} 0.039$ \\
\hline SD & 17905.20 & 46314.23 & & 36817.45316 & 16102.4601 & \\
\hline CSF $n=15$ & $\begin{array}{l}\mathrm{ClS} \\
N=10\end{array}$ & $\begin{array}{l}\text { 1st attack MS } \\
N=5\end{array}$ & $p$-value & $\begin{array}{l}\text { MS convertors } \\
N=6\end{array}$ & $\begin{array}{l}\text { MS non-convertors } \\
N=4\end{array}$ & $p$-value \\
\hline $\operatorname{lgG}$ index & $8(80 \%)$ & $3(60 \%)$ & 0.40 & $6(100 \%)$ & $2(50 \%)$ & 0.74 \\
\hline Positive & 7 (70\%) & $3(60 \%)$ & 0.698 & $6(100 \%)$ & $1(25 \%)$ & ${ }^{*} 0.033$ \\
\hline
\end{tabular}


Table 3 Ultrasonography assessment and MRI results of all studied groups

\begin{tabular}{|c|c|c|c|c|c|c|c|c|}
\hline & & & \multirow{2}{*}{$\begin{array}{l}\text { Group } 1 \\
\text { CIS } \\
N=22\end{array}$} & \multirow{2}{*}{$\begin{array}{l}\text { Group } 2 \\
1 \text { st attack MS } \\
N=20\end{array}$} & \multirow[b]{2}{*}{$p$-value } & \multicolumn{2}{|l|}{ CIS group } & \multirow[b]{2}{*}{$p$-value } \\
\hline & & & & & & $\begin{array}{l}\text { MS convertors } \\
N=9\end{array}$ & $\begin{array}{l}\text { MS non-convertors } \\
N=13\end{array}$ & \\
\hline \multicolumn{9}{|l|}{ Brain imaging } \\
\hline \multirow{2}{*}{\multicolumn{2}{|c|}{ T2 MRI brain lesions }} & Typical & $11(50 \%)$ & $20(100 \%)$ & $*^{*} 0.003$ & $7(77.8 \%)$ & $4(30.8 \%)$ & 0.08 \\
\hline & & Atypical & $11(50 \%)$ & $0(0 \%)$ & & $2(22.2 \%)$ & $9(69.2 \%)$ & \\
\hline \multicolumn{2}{|l|}{ T1 black holes } & $\geq 1$ & $5(22.7 \%)$ & $15(75 \%)$ & ${ }^{*} 0.004$ & $3(33.3 \%)$ & $2(15.4 \%)$ & 0.502 \\
\hline \multicolumn{2}{|c|}{ Total number of lesions } & (mean $\pm \mathrm{SD}$ ) & $4.86 \pm 4.20$ & $9.65 \pm 3.79$ & $*^{*} 0.001$ & $6.22 \pm 4.89$ & $3.92 \pm 3.55$ & 0.357 \\
\hline \multicolumn{3}{|c|}{ Patients with MRI enhancing lesions } & $5(22.7 \%)$ & $15(75 \%)$ & ${ }^{*} 0.001$ & $3(33.3 \%)$ & $2(15.4 \%)$ & 0.841 \\
\hline \multirow{2}{*}{\multicolumn{2}{|c|}{ Substantia nigra echogenicity }} & Normal & $11(55 \%)$ & $15(75 \%)$ & 0.2 & $2(25 \%)$ & $9(75 \%)$ & 0.06 \\
\hline & & Hypoechogenic & $9(45 \%)$ & $5(25 \%)$ & & $6(75 \%)$ & $3(25 \%)$ & \\
\hline \multirow[t]{4}{*}{ Ventricular diameter } & 3rd ventricle & Normal (\%) & $14(66.7 \%)$ & $17(85 \%)$ & 0.277 & $5(62.5 \%)$ & $9(69.2 \%)$ & 1 \\
\hline & & Dilated (\%) & 7 (33.3\%) & $3(15 \%)$ & & $3(37.5 \%)$ & 4 (30.8\%) & \\
\hline & Frontal horn & Median $(\mathrm{cm})$ & 0.19 & 0.22 & 0.916 & 0.19 & 0.19 & 0.600 \\
\hline & & Dilated (\%) & $5(25 \%)$ & $2(10.5 \%)$ & 0.661 & $3(37.5 \%)$ & 2 (16.7\%) & 0.255 \\
\hline
\end{tabular}

MS lesions were higher in the MS convertor group, with near significance value. This is close to previous studies, which showed that the risk of conversion to MS after CIS increased with increased baseline T2 lesion load, and was more in patients with typical lesions fulfilling 3-4 Barkhof criteria in baseline scan [19, 20].

We assessed cognitive dysfunction as a manifestation related to axonal loss and brain atrophy [21], and since several studies reported that early cognitive affection in CIS can predict short time for conversion to MS, suggesting that it may represent a sensitive marker of more severe changes within the lesions [3, 22], our results did not support such findings as we did not detect significant difference in the degree of cognitive affection between the MS group and CIS patients and MS converter group versus MS non-converter.

We further assessed neurodegeneration by the detection of markers of brain atrophy using parenchymatous transcranial sonography. The ventricular diameter for 3rd ventricle and frontal horn of lateral ventricle were measured; however, we found no significant difference among the studied groups. Hyperechogenicity of SN that was related in previous studies to changes of brain iron

Table 4 Logistic regression to detect predictors of early conversion to MS

\begin{tabular}{lllll}
\hline Independent predictors & $\boldsymbol{P}$ value & OR & \multicolumn{2}{l}{$\mathbf{9 5 \%} \mathrm{Cl}$} \\
\cline { 4 - 6 } & & & Lower & Upper \\
\hline MoCA test & .562 & 1.079 & .834 & 1.396 \\
CHI3L1 assay & .301 & 1.000 & 1.000 & 1.000 \\
Ultrasound ventricular diameter & .834 & .418 & .000 & 1439.470 \\
Total number of T2 lesions & $* 0.003$ & 1.33 & 1.105 & 1.604 \\
\hline
\end{tabular}

metabolism and correlated with future progress of MS $[6,23]$ was not detected in any of our subjects; SN was either normal or hypoechoic. This discrepancy can be explained by the type of patients studied as all our subjects were in the early course of the disease (either CIS or first attack of MS), they were younger (mean age $29.41 \pm 6.94$ ), with milder form (EDSS range $=1-2$ ) while the previous studies included older patients (mean age range $=37.9-51.5$ years) with relapsing-remitting $\mathrm{MS}$ (RRMS), secondary progressive MS (SPMS), and primary progressive MS, and since both studies found a significant correlation between the area of the substantia nigra, and the age of patients, the duration of the illness, EDSS score, and the number of relapses, we can conclude that such sonographic findings may be not of significance in the early course of the disease.

Logistic regression was done to detect independent predictors of conversion to MS; it showed that high baseline T2 lesion load was the only significant predictor for conversion to MS after CIS which goes with several previous studies $[19,20]$.

\section{Conclusion}

In view of the aim of the study to detect and compare different markers related to the pathological processes underlying demyelination and to assess their prognostic value, we found that none of the markers of neurodegeneration tested (cognitive affection, T1 black holes and sonographic parameters) served as predictors for MS conversion while within markers of inflammation (elevated OCB, chitinase level, high baseline T2 lesion and enhanced MRI lesions) only heavy T2 lesion load showed had a significant prognostic value compared to the other markers. 


\section{Abbreviations}

ANA: Antinuclear antibody; ANCA: Antineutrophil cytoplasmic antibodies; BG: Basal ganglia; CDMS: Clinically definite multiple sclerosis; CHI3L1: Chitinase 3-like 1; CIS: Clinically isolated syndrome;

CSF: Cerebrospinal fluid; DMT: Disease-modifying therapy; EDSS: Expanded Disability Status Scale; MoCA: Montreal Cognitive Assessment; MRI: Magnetic resonance imaging; MS: Multiple sclerosis; OCB: Oligoclonal bands; RRMS: Relapsing-remitting MS; SN: Substantia nigra; SPMS: Secondary progressive MS; TCS: Transcranial sonography

\section{Acknowledgements}

The authors acknowledge Prof. Dr. Ahmed Abo Mosa, for his valuable instructions with the initial design of this study.

\section{Authors' contributions}

All authors contributed to the research idea. AA and EA contributed to the data collection. $\mathrm{HH}$, RS and AA analyzed the data along with NF and RS who interpreted the data. Further NF, HH and RS completed the first draft of the article. All authors were involved in revising it critically for important intellectual content, and all authors approved the final version to be published.

\section{Funding}

There is no source of funding for this research

\section{Availability of data and materials}

All datasets generated and analyzed during the current study are not publicly available, but are available by reasonable request from the corresponding author.

\section{Declarations}

\section{Ethics approval and consent to participate}

All procedures performed in the study were in accordance with the ethical standards of the institutional research committee and with the 1964 Helsinki Declaration and its later amendments. The study was ethically approved by research committee and reviewed by the Faculty of Medicine Cairo University board (clinical trial number NCT02854384) registration date January 2015; committee's reference number is not applicable. Written informed consent was obtained from all participants involved in this investigation prior to the conduct of any study-related activities

\section{Consent for publication}

Not applicable

\section{Competing interests}

The authors declare that they have no competing interests (financial and non-financial),

\section{Author details}

${ }^{1}$ Neurology Department, Cairo University, Cairo, Egypt. ²Department of Clinical \& Chemical Pathology, Cairo University, Cairo, Egypt.

\section{Received: 28 July 2020 Accepted: 13 July 2021}

Published online: 05 October 2021

\section{References}

1. Marcus JF, Waubant EL. Updates on clinically isolated syndrome and diagnostic criteria for multiple sclerosis. The Neurohospitalist. 2013;3(2):6580. https://doi.org/10.1177/1941874412457183.

2. Sturm D, Gurevitz SL, Turner A. Multiple sclerosis: a review of the disease and treatment options. Consult Pharm. 2014;29(7):469-79. https://doi.org/1 0.4140/TCP.n.2014.469.

3. Charvet LE. The Montreal Cognitive Assessment (MoCA) in multiple sclerosis: relation to clinical features. J Mult Scler. 2015;2:135.

4. Hynčicová E, Vyhnálek M, Kalina A, Martinkovič L, Nikolai T, Lisý J, et al. Cognitive impairment and structural brain changes in patients with clinically isolated syndrome at high risk for multiple sclerosis. J Neurol. 2017;264(3): 482-93. https://doi.org/10.1007/s00415-016-8368-9.
5. Muller M, Muller A, Kotter K, Osterreich M, Stellmes P. Width of the third ventricle as assessed by transcranial sonography is associated with future relapses in mild multiple sclerosis. J Neurol Neurosci. 2016;7:S3.

6. Walter U, Wagner S, Horowski S, Benecke R, Zettl UK. Transcranial brain sonography findings predict disease progression in multiple sclerosis. Neurology. 2009;73(13):1010-7. https://doi.org/10.1212/WNL.0b013e3181 b8a9f8.

7. Huss AM, Halbgebauer S, Öckl P, Trebst C, Spreer A, Borisow N, et al. Importance of cerebrospinal fluid analysis in the era of McDonald 2010 criteria: a German-Austrian retrospective multicenter study in patients with a clinically isolated syndrome. J Neurol. 2016;263(12):2499-504. https://doi. org/10.1007/s00415-016-8302-1.

8. Comabella M, Fernández M, Martin R, Rivera-Vallvé S, Borrás E, Chiva $C$, et al. Cerebrospinal fluid chitinase 3-like 1 levels are associated with conversion to multiple sclerosis. Brain. 2010;133(Pt(4)):1082-93.

9. Bonneh-Barkay D, Bissel SJ, Kofler J, Starkey A, Wang G, Wiley CA. Astrocyte and macrophage regulation of YKL-40 expression and cellular response in neuroinflammation. Brain Pathol. 2012;22(4):530-46. https://doi.org/10.1111/ j.1750-3639.2011.00550.x.

10. Canto E, Tintore M, Villar LM, Costa C, Nurtdinov R, Alvarez-Cermeno JC, et al. Chitinase 3-like 1: Prognostic biomarker in clinically isolated syndromes. Brain. 2015;138(4):918-31. https://doi.org/10.1093/brain/awv017.

11. Hinsinger G, Galéotti N, Nabholz N, Urbach S, Rigau V, Demattei C, et al. Chitinase 3-like proteins as diagnostic and prognostic biomarkers of multiple sclerosis. Mult Scler. 2015;21(10):1251-61. https://doi.org/10.1177/13 52458514561906

12. Odenthal C, Coulthard A. The prognostic utility of MRI in clinically isolated syndrome: a literature review. Am J Neuroradiol. 2015;36(3):425-31. https:// doi.org/10.3174/ajnr.A3954.

13. Kurtzke JF. Rating neurologic impairment in multiple sclerosis: an expanded disability status scale (EDSS). Neurology. 1983;33(11):1444-52. https://doi. org/10.1212/WNL.33.11.1444.

14. Shrank WH, Choudhry NK, Liberman JN, Brennan TA. The use of generic drugs in prevention of chronic disease is far more cost-effective than thought, and may save money. Health Aff. 2011;30(7):1351-7. https://doi. org/10.1377/hlthaff.2010.0431.

15. Saleh AA, Alkholy RSAEHA, Khalaf OO, Sabry NA, Amer $\mathrm{H}$, El-Jaafary $\mathrm{S}$, et al. Validation of Montreal Cognitive Assessment-Basic in a sample of elderly Egyptians with neurocognitive disorders. Aging Ment Health. 2019;9:1-7.

16. Hamdy SM, Abdel-Naseer M, Shalaby NM, Eelmazny AN, Nemr AA, Hassan A, et al. Characteristics and predictors of progression in an Egyptian multiple sclerosis cohort: a multicenter registry study. Neuropsychiatr Dis Treat. 2017; 13:1895-903. https://doi.org/10.2147/NDT.S140869.

17. Polman CH, Reingold SC, Banwell B, Clanet M, Cohen JA, Filippi M, et al. Diagnostic criteria for multiple sclerosis: 2010 Revisions to the McDonald criteria. Ann Neurol. 2011;69(2):292-302. https://doi.org/10.1002/ana.22366.

18. Schwenkenbecher P, Sarikidi A, Bönig L, Wurster U, Bronzlik P, Sühs KW, et al. Clinically isolated syndrome according to mcdonald 2010: Intrathecal IgG synthesis still predictive for conversion to multiple sclerosis. Int J Mol Sci. 2017;18(10):2061. https://doi.org/10.3390/ijms18102061.

19. Alroughani R, Al Hashel J, Lamdhade S, Ahmed SF. Predictors of conversion to multiple sclerosis in patients with clinical isolated syndrome using the 2010 Revised McDonald criteria. ISRN Neurol. 2012.

20. Ruet A, Arrambide G, Brochet B, Auger C, Simon E, Rovira À, et al. Early predictors of multiple sclerosis after a typical clinically isolated syndrome. Mult Scler J. 2014;20(13):721-6.

21. De Stefano N, Narayanan S, Francis GS, Arnaoutelis R, Tartaglia MC, Antel JP, et al. Evidence of axonal damage in the early stages of multiple sclerosis and its relevance to disability. Arch Neurol. 2001;58(1):65-70. https://doi. org/10.1001/archneur.58.1.65.

22. Zipoli V, Goretti B, Hakiki B, Siracusa G, Sorbi S, Portaccio E, et al. Cognitive impairment predicts conversion to multiple sclerosis in clinically isolated syndromes. Mult Scler. 2010;16(1):62-7. https://doi.org/10.1177/13524585093 50311.

23. Puz P, Lasek-Bal A, Radecka P. Transcranial sonography of subcortical structures in patients with multiple sclerosis. Acta Neurol Scand. 2017;136(1): 24-30. https://doi.org/10.1111/ane.12689.

\section{Publisher's Note}

Springer Nature remains neutral with regard to jurisdictional claims in published maps and institutional affiliations. 